\section{OP30 LIFETIME SEVERE AFFECTIVE SYMPTOMS AND SUBSEQUENT MENTAL STATUS: OVER 50 YEARS OF FOLLOW-UP IN THE 1946 BRITISH BIRTH COHORT STUDY}

SN James*, N Sharma, C O'Hare, D Kuh, M Richards. MRC Unit for Lifelong Health and Ageing, at University College London, London, UK

\subsection{6/jech-2017-SSMAbstracts.30}

Background Affective problems are common, involving one in five individuals across their lifetime. Affective problems increase the risk for subsequent dementia and cognitive impairment, but the nature of this association is not yet clearly understood. Symptom severity is known to raise the risk, but it is unclear whether this risk is cumulative. We investigated prospective associations between lifetime severe affective symptoms and subsequent mental status and cognitive decline in a population-based sample aged 69 years, still mostly free of dementia, controlling for potential confounders. Methods Complete data from1059 study members from the Medical Research Council National Survey of Health and Development (NSHD, the 1946British birth cohort) were analysed. Prospectively-assessed affective symptoms at ages 13-15, $36,43,53,60-64$ and 69 were used to generate severe (caselevel) binary indicators of affective symptoms at each age which were then summed to give a cumulative score and operationalised as 0 (never case-level), 1 (case-level at only one assessment), or 2 (case-level at two or more assessments). Mental status was assessed using the Addenbrooke's Cognitive Examination (ACE-III) at age 69. Measures of short-term memory, letter search speed and accuracy were assessed at ages 60-64 and 69. Linear regression models were conducted to investigate whether case-level affective symptoms were associated with lower mental status and cognition at age 69, conditional on cognition at age 60-64, and adjusting for gender, childhood cognition, childhood and midlife social class and educational attainment.

Results Compared with those with no evidence of affective problems, those with case-level symptoms at only one assessment did not differ in any of the cognitive measures, before and after adjustments. However, those with case-level affective symptoms at two or more assessments had a lower ACE-III score $(\beta=-0.88,95 \% \mathrm{CI}=-1.66,-0.10)$, and greater decline in short-term memory $(\beta=-0.56,95 \% \mathrm{CI}=-1.21,-0.09)$, search speed $(\beta=-11.11,95 \% \mathrm{CI}=-19.59,-2.64)$, and search accuracy $(\beta=-0.71,95 \% \mathrm{CI}=-1.29,-0.13)$ between ages 60 and 69 , before and after adjustments.

Conclusion Overall, our findings show that those with recurrent severe affective problems are more likely to have poorer mental status and faster cognitive decline. One limitation is that there may be additional confounding factors that we have been unable to account for, including the potential for reverse causality. However, this is still one of the largest prospective studies to show the association between lifetime severe affective symptoms and subsequent cognitive function. Further follow-up will determine what proportion with severe recurrent symptoms develop clinical dementia.

\section{OP31 CHARACTERISING LONGITUDINAL PATTERNS OF BACK PAIN ACROSS ADULTHOOD IN THE 1946 BRITISH BIRTH COHORT}

S Muthuri ${ }^{*}$, D Kuh, R Cooper. MRC Unit for Lifelong Health and Ageing, University College London, London, UK

\subsection{6/jech-2017-SSMAbstracts.31}

Background Back pain is the most common musculoskeletal condition worldwide and a leading cause of years lived with disability. It affects people of all ages and is a major cause of activity limitation. Back pain is often a recurrent or long-term condition; however, most previous longitudinal studies have short follow-up periods or limited follow-up points over a long timeframe and, have sampled populations from workplace or clinical settings. We aimed to characterise the long-term profiles of back pain over adulthood and examine whether early life factors are associated with these profiles independent of established adult risk factors.

Methods Data were drawn from a nationally representative British birth cohort, the MRC National Survey of Health and Development. For 3271 participants with at least three assessments of back pain, a longitudinal latent variable analysis was conducted on binary outcomes of back pain at ages 31,36 , 43, 53, 60-64 and 68 using Mplus v 6.12. Derived class posterior probabilities were exported to Stata v14.1 and multinomial logistic regression models with probability weighting were used to examine associations between selected childhood factors (including height, body mass index, serious illness, abdominal pain, emotional and conduct problems, father's occupational class, mother's education, parental divorce and parental health) and class membership. Adjustments were made for sex and adult risk factors.

Results Four profiles of back pain were identified: no or occasional pain (54.6\% women: $60.8 \%$ men), early-adulthood only (16.6\%: 15.5\%), mid-adulthood onset (18.0\%: 15.7\%) and persistent (10.8\%: 7.9\%) pain. No or occasional pain profile was treated as the referent category in subsequent analyses.

In sex-adjusted models, greater height at age 7 was associated with a higher likelihood of early-adulthood only (Relative-Risk Ratio (RRR) per $1 \mathrm{~cm}$ increase in height $=1.03[95 \%$ CI: 1.01-1.05]) and persistent pain $(\mathrm{RRR}=1.04$ [95\%CI: 1.02-1.07]). Other factors associated with increased risk of persistent pain were manual father's occupational class, severe conduct problems, parental divorce, abdominal pain and average-poor parental health. The latter two factors were also associated with an increased likelihood of mid-adulthood onset pain. In models which included mutual adjustment for other early life factors and adult risk factors, only height at age 7 remained independently associated with early-adulthood only and persistent pain.

Conclusion These findings suggest that a range of factors in early life, most importantly height, are risk factors for longterm experience of back pain across adulthood; therefore interventions that aim to alleviate the burden of back pain may need to start early. 